\title{
AVALIAÇÃO PÓS-TEMPESTADE DA ARBORIZAÇÃO DO CAMPUS DA UNIVERSIDADE DO ESTADO DE SANTA CATARINA, LAGES-SC
}

\author{
Pamela Moser ${ }^{1,3}$, Ana Carolina da Silva ${ }^{2}$, Pedro Higuchi ${ }^{2}$, Érica Moraes dos Santos ${ }^{1}$, \\ Verônica Schmitz ${ }^{1}$
}

(recebido em 03.03.2010 e aceito para publicação em 10.06.2010)

\section{RESUMO}

As árvores nas cidades trazem benefícios como melhorias na paisagem, proporcionando ambientes que favorecem o convívio social e a relação do homem com a natureza, assim como o equilíbrio entre as áreas verdes e o concreto encontrado nas cidades. Porém, uma arborização com a utilização de espécies inadequadas ao espaço urbano, como as de porte muito grande ou susceptível aos ventos, pode trazer problemas, como a queda de árvores ou de grandes galhos, que causam prejuízos materiais e acidentes. Este estudo foi realizado no Centro de Ciências Agroveterinárias (CAV) da Universidade do Estado de Santa Catarina (UDESC) em Lages, SC, e objetivou conhecer a composição florística da arborização urbana do campus e avaliar os danos causados às árvores após uma tempestade com ventos intensos. A arborização do CAV-UDESC é composta por 791 indivíduos distribuídos em 39 espécies nativas e exóticas, sendo que os gêneros com maior número de indivíduos foram Eucalyptus e Pinus. Algumas espécies foram mais danificadas pela ação das rajadas intensas de ventos, entre elas Cupressus lusitanico, recomendando-se evitar a sua utilização na arborização urbana.

Palavras-chave: levantamento florístico, quebra de árvores, vento.

\footnotetext{
1. Graduandos do curso de Engenharia Florestal da Universidade do Estado de Santa Catarina. Lages/SC.

2. Professores do Departamento de Engenharia Florestal, Universidade do Estado de Santa Catarina, Av. Luiz de Camões, 2090, Bairro Conta Dinheiro, Lages, SC, CEP: 88520-000.

3.E-mail para contato: pamelamozer@yahoo.com.br
} 


\section{TREES ASSESSMENT AFTER A WIND STORM IN SANTA CATARINA STATE UNIVERSITY CAMPUS, LAGES-SC}

\section{ABSTRACT}

Urban trees provide benefits such as landscape improvement providing environments that inspire social contact and the relationship between man and nature, as well as the balance between green and concrete areas in the city. However, the use of inappropriate species in urban areas, such as those trees that can reach large sizes or susceptible to damage due to winds, can cause problems as fallen of trees and large branches, causing damage and accidents. This study was conducted at the Center of Agroveterinary Science (CAV) in Santa Catarina State University (UDESC) in Lages, SC. The aim of this study was to identify urban forest and floristic composition of the campus and to assess the damage caused to the trees after a wind storm. The CAV-UDESC urban forest is composed of 791 individuals distributed in 39 native and exotic species. The genera with the highest number of individuals were Eucalyptus and Pinus. Some species were more damaged by the action of intense wind gusts, including Cupressus lusitanica. Thus, wind-damage-prone species should be avoided in urban forest.

Keywords: Floristic survey, fallen trees, wind 


\section{INTRODUÇÃO}

As cidades brasileiras enfrentam sérios problemas acarretados pelo mau planejamento urbano que ocorreram nas últimas décadas. Este fato se agrava ao considerar o acelerado desenvolvimento sem consciência ambiental, que vem gerando modificações negativas no meio ambiente, como as mudanças climáticas. Por ser responsável por melhorias na paisagem (BRADSHAW et al., 1995) e mitigar efeitos ambientais negativos que ocorrem em função da expansão urbana, a presença das árvores nas cidades é importante, de forma que o planejamento da arborização urbana se tornou uma prática fundamental.

Os indivíduos arbóreos são importantes para o desenvolvimento social, não somente quando se trata de questões ambientais e econômicas, mas também estéticas, históricas e espirituais, pelos diversos benefícios diretos e indiretos proporcionados (SETH, 2004). Segundo Leal et al. (2008), as árvores atenuam linhas arquiteturais, aliviam a monotonia do pavimento e da alvenaria, reduzem a proporção entre espaços construídos e o homem no meio urbano, e acrescentam dinamismo à paisagem pelos seus aspectos fenológicos. A importância desta atividade para a conservação da natureza ocorre devido a vários fatores, como, por exemplo, as árvores servirem como fonte de alimento para a fauna silvestre e poderem seqüestrar carbono atmosférico durante seu crescimento e acúmulo de biomassa.

No entanto, a falta de planejamento da arborização urbana pode provocar danos materiais e humanos. Esses danos são causados devido ao conflito entre as árvores e a infra-estrutura da cidade, como exemplo o levantamento das calçadas ocasionado pelo desenvolvimento das raízes (RANDRUP et al., 2003), e a queda de ramos ou de árvores inteiras em condições de fortes ventos, muitas vezes associados a elevados níveis de precipitação (Oliveira e Lopes, 2007). A queda de árvores ou de partes dela é uma das principais causas de ferimentos em pessoas - podendo levar até a morte - na presença de condições extremas de ventos (SARAIVA, 1983 e OFDA/CRED, 2007, citados por OLIVEIRA e LOPES, 2007).

Alguns dos eventos que têm sido observados com maior freqüência nos últimos anos, atingindo freqüentemente as Regiões Sul e Sudeste do Brasil, são as tempestades com fortes rajadas de vento, tornados e ciclones, que ocorrem principalmente durante o verão. Nos noticiários são relatados casos de quedas de árvores de grande porte que resultam nestas perdas materiais e, ou, humanas. Um dos fatores que contribuem para essas quedas é a utilização de espécies inadequadas ao espaço urbano, como espécies de 
grande porte, que quando caem causam muitos estragos, e espécies susceptíveis aos ventos, que possuem raízes superficiais, caindo facilmente, ou com galhos que quebram quando submetido ao vento forte. Segundo Oliveira e Lopes (2007), a idade, a dimensão, a densidade da folhagem, a largura do fuste e a densidade da madeira das árvores, além de fatores como a construção de edifícios e pavimentos que causam a diminuição da camada superficial e a compactação do solo, diminuindo o volume de solo disponível para o crescimento das raízes, são fatores que podem contribuir para a queda destas árvores.

O presente estudo se concentrou no levantamento das árvores danificadas e nas que permaneceram sem danos no campus da Universidade Estadual de Santa Catarina, após tempestade de ventos, objetivando: i) conhecer a composição florística das espécies arbóreas localizadas no campus e ii) avaliar os danos causados na arborização após a ocorrência dessa tempestade.

\section{MATERIAIS E MÉTODOS}

O estudo foi realizado na cidade de Lages, SC, nos limites do Centro de Ciências Agroveterinárias (CAV), campus da Universidade do Estado de Santa Catarina (UDESC), situado geograficamente nas coordenadas $27^{\circ} 48^{\prime} 58^{\prime \prime S}$ e 50¹9'34" O, com altitude de $940 \mathrm{~m}$ (IBGE, 2009).

No dia 2 de dezembro de 2009 ocorreu uma tempestade com rajadas de vento de 80 a $100 \mathrm{~km} / \mathrm{h}$ em Lages, SC (SILVA, 2010). Na cidade e no campus do Centro de Ciências Agroveterinárias (CAV) da Universidade do Estado de Santa Catarina (UDESC), localizado em Lages, foram observados diversos danos materiais e a queda de galhos e árvores. Em Urubici, que encontra se a $32 \mathrm{~km}$ de Lages, no mesmo dia o INMET registrou rajadas de ventos de $125 \mathrm{~km} / \mathrm{h}$ (SILVA, 2010).

Em Lages foram observados diversos danos materiais, com a queda de várias árvores, destelhamento de casas, quebra de vidros de janelas e outros. No CAV-UDESC foi constatada a queda de árvores e quebra de galhos, causando danos em automóveis e construções. Foi realizado o censo das árvores do campus com DAP (diâmetro medido à altura do peito) $\geq 4,8 \mathrm{~cm}$ que sofreram quedas e quebras de caules após esta tempestade $\mathrm{e}$ daquelas que permaneceram sem danos. Essas árvores foram identificadas e medidas (altura e DAP - diâmetro medido à altura do peito). Foi quantificado o número de indivíduos 
por espécie que sofreu diferentes classes de danos (árvores arrancadas ou quebradas). Por meio do teste qui-quadrado, realizado no programa $R$ ( $R$ DEVELOPMENT CORE TEAM, 2009), foi verificado se os danos ocorreram de forma aleatória entre as espécies ou se houve susceptibilidade de espécies aos danos. Nesta análise foram obtidos os valores esperados de árvores danificadas, para uma situação de total aleatoriedade para ocorrência de danos nas espécies estudadas. Para uma determinada espécie, se o valor observado de árvores danificadas foi maior que esperado para uma situação de aleatoriedade, esta espécie foi considerada como suscetível aos danos provocados pelo vento.

\section{RESULTADOS E DISCUSSÃO}

Foram identificados e mensurados 791 indivíduos arbóreos distribuídos em 23 famílias botânicas e pertencentes a 39 espécies (Tabela 1). As famílias que apresentaram maior riqueza de espécies foram Bignoniaceae (quatro espécies) e Fabaceae (cinco espécies), sendo a primeira representada pelos ipês e jacarandá mimoso, enquanto o outro grupo foi formado por bracatinga, canafístula, sena, tipuana e rabo-de-bugio. Pode-se observar que as árvores que compõe a arborização do CAV/UDESC estão distribuídas ao longo de passeios, proporcionando sombra e interligando as principais edificações. Algumas árvores estão distribuídas em grupos, criando um ambiente favorável para o estudo, o convívio social, a contemplação e o contato com a natureza.

TABELA 1 - Espécies arbóreas do Campus da UDESC e danos ocasionados nestas pelos ventos. $\mathrm{DAP}=$ diâmetro medido à altura do peito médio; $\mathrm{H}=$ altura média; $\mathrm{N}=$ número de indivíduos; ND = número de árvores danificadas, sendo estas arrancadas $\left({ }^{*}\right)$ ou quebradas $\left.{ }^{(*}\right) ; \mathrm{NE}=$ número esperado de árvores danificadas, pelo teste qui-quadrado $(p=0,0023)$

\begin{tabular}{|c|c|c|c|c|c|c|c|}
\hline Família/espécie & $\begin{array}{l}\text { Nome } \\
\text { popular }\end{array}$ & Origem & $\begin{array}{l}\text { DAP } \\
(\mathrm{cm})\end{array}$ & $\begin{array}{l}\mathbf{H} \\
(\mathrm{m})\end{array}$ & $\mathbf{N}$ & ND & NE \\
\hline \multicolumn{8}{|l|}{ Anacardiaceae } \\
\hline Schinus molle L. & $\begin{array}{l}\text { aroeira } \\
\text { salsa }\end{array}$ & $\begin{array}{l}\text { Colômbia, Peru, Bolívia, } \\
\text { Paraguai, Argentina e } \text { Uruguai }^{\mathrm{a}}\end{array}$ & 15,1 & 5,5 & 1 & 0 & 0,04 \\
\hline $\begin{array}{l}\text { Schinus terebinthifolius } \\
\text { Raddi }\end{array}$ & $\begin{array}{l}\text { aroeira } \\
\text { vermelha }\end{array}$ & $\begin{array}{l}\text { Brasil, Uruguai e } \\
\text { Argentina }^{\mathrm{a}}\end{array}$ & 17,5 & 6,9 & 53 & $1^{* *}$ & 2,28 \\
\hline Apocynaceae & & & & & & & \\
\hline $\begin{array}{l}\text { Nerium oleander L. } \\
\text { Araucariaceae }\end{array}$ & espirradeira & Europa e África $^{\mathrm{b}}$ & 12,4 & 5,6 & 2 & 0 & 0,09 \\
\hline $\begin{array}{l}\text { Araucaria angustifolia } \\
\text { (Bertol.) Kuntze } \\
\text { Arecaceae }\end{array}$ & $\begin{array}{l}\text { pinheiro } \\
\text { brasileiro }\end{array}$ & Brasil e Argentina $^{a}$ & 29,7 & 10,5 & 21 & $1^{*}+1^{* *}$ & 0,90 \\
\hline Butia eriospatha (Mart. ex & butiá & Brasil $^{\mathrm{a}}$ & 50,0 & 6,8 & 3 & 0 & 0,13 \\
\hline
\end{tabular}


Drude) Becc.

Syagrus romanzoffiana

(Cham.) Glassman

Bignoniaceae

Handroanthus albus

(Cham.) Mattos

Handroanthus

chrysotrichus (Mart. ex

A.DC.) Mattos

Handroanthus heptaphyllus ipê roxo

(Martius) Mattos

Jacaranda mimosifolia

D.Don.

\section{Cupressaceae}

Chamaecyparis obtusa

(Siebold \& Zucc.) Endl.

Cupressus lusitanica Mill.

Thuja orientalis L.

Euphorbiaceae

Sapium glandulosum (L.) leiteiro

Morong

\section{Fabaceae}

Lonchocarpus campestris

Mart. ex Benth.

Mimosa scabrella Benth.

Peltophorum dubium

(Spreng.) Taub.

Senna pendula (Willd.)

H.S.Irwin \& Barneby

Tipuana tipu (Benth.)

Kuntze

Fagaceae
Castanea sativa Mill.
Quercus robur L.
Juglandaceae
Pterocarya fraxinifolia
(Lam.) Spach.
Lauraceae
Ocotea puberula (Rich.)
Nees

Nees

Ocotea pulchella Mart.

Lythraceae

Lagerstroemia indica L.

Magnoliaceae

Magnolia champaca (L.)

Baill.

rabo-de-

bugio

bracatinga

canafístula

ipê amarelo

ipê amarelo

Argentina $^{a}$

Brasil, Uruguai, Paraguai

e Argentina $^{a}$

jacarandá

mimoso

cipreste

dourado

cipreste

português

tuia

Brasil e Argentina $^{a}$

Brasil, Uruguai, Paraguai,

Argentina e Bolívia ${ }^{a}$

Argentina, Bolívia e

Paraguai $^{\mathrm{b}}$

Japão ${ }^{b}$

México, Guatemala, El

Salvador e Honduras ${ }^{\mathrm{b}}$

China e Ásia Oriental ${ }^{b}$

Brasil, Uruguai, Argentina,

Paraguai, Bolívia,

Floresta Amazônica à

América Central $^{a}$

Brasil, Argentina e

Paraguai $^{\mathrm{a}}$

Brasil $^{a}$

Brasil, Uruguai, Argentina,

Paraguai, Bolívia e

Caribe $^{a}$

cigarreira Brasil, Argentina,

Paraguai, Bolívia,

Floresta Amazônica à

América Central $^{a}$

tipuana Bolívia e Argentina ${ }^{\mathrm{b}}$

castanha
portuguesa
carvalho

Europa, África e China ${ }^{\mathrm{b}}$

Europa, África e Ásia ${ }^{\mathrm{b}}$

vermelho

pterocária

Cáucaso $^{b}$

canela

guaica

Brasil, Argentina,

Paraguai, Bolívia,

Floresta Amazônica à

América Central $^{a}$

canela

lageana

Brasil, Argentina e

Paraguai $^{\mathrm{a}}$

extremosa índia ${ }^{b}$

magnólia Índia e Himalaia ${ }^{\text {b }}$

amarela $\begin{array}{lllll}34,4 & 6,0 & 1 & 0 & 0,04\end{array}$

$\begin{array}{lllll}25,2 & 8,2 & 13 & 0 & 0,52\end{array}$

$\begin{array}{lllll}19,2 & 4,7 & 3 & 0 & 0,13\end{array}$

$\begin{array}{lllll}14,3 & 7,3 & 9 & 0 & 0,39\end{array}$

$\begin{array}{lllll}34,1 & 8,7 & 1 & 0 & 0,04\end{array}$

$\begin{array}{lllll}13,6 & 7,8 & 3 & 0 & 0,13\end{array}$

$27,5 \quad 10,6 \quad 57 \quad 5^{*}+2^{* *} \quad 2,45$

$\begin{array}{lllll}20,5 & 9,2 & 2 & 0 & 0,09\end{array}$

$\begin{array}{lllll}10,0 & 4,7 & 1 & 0 & 0,04\end{array}$

$\begin{array}{lllll}9,0 & 4,7 & 19 & 3^{*} & 0,82\end{array}$

$\begin{array}{lllll}22,4 & 10,0 & 1 & 0 & 0,04\end{array}$

$\begin{array}{lllll}21,0 & 7,2 & 2 & 1^{*} & 0,09\end{array}$

$18,7 \quad 7,1 \quad 7 \quad 1^{*}+2^{* *} \quad 0,30$

$\begin{array}{lllll}87,7 & 7,0 & 1 & 0 & 0,04\end{array}$

$\begin{array}{lllll}94,9 & 15,0 & 1 & 0 & 0,04\end{array}$

$\begin{array}{lllll}7,3 & 4,0 & 1 & 0 & 0,04\end{array}$

$\begin{array}{lllll}23,9 & 8,5 & 3 & 0 & 0,13\end{array}$

$\begin{array}{lllll}23,7 & 6,0 & 3 & 0 & 0,13\end{array}$

$\begin{array}{lllll}8,6 & 6,2 & 1 & 0 & 0,04\end{array}$

$\begin{array}{lllll}14,6 & 6,0 & 1 & 0 & 0,04\end{array}$

Meliaceae 


\begin{tabular}{|c|c|c|c|c|c|c|c|}
\hline Cedrela fissilis Vell. & cedro & $\begin{array}{l}\text { Brasil, Uruguai, Argentina, } \\
\text { Paraguai, Bolívia, } \\
\text { Floresta amazônica à }^{\text {América Central }}{ }^{a}\end{array}$ & 23,5 & 8,0 & 6 & 0 & 0,26 \\
\hline $\begin{array}{l}\text { Melia azedarach L. } \\
\text { Myrtaceae }\end{array}$ & cinamomo & Índia e China ${ }^{b}$ & 53,6 & 10,0 & 2 & 0 & 0,09 \\
\hline Callistemon viminalis Sol. & $\begin{array}{l}\text { escova de } \\
\text { garrafa }\end{array}$ & Austrália $^{b}$ & 11,3 & 5,0 & 2 & 0 & 0,09 \\
\hline Eucalyptus spp. & eucalipto & $\begin{array}{l}\text { Oceania e Sudeste da } \\
\text { Ásiab }^{\text {b }}\end{array}$ & 46,2 & 22,4 & 319 & $6^{*}+3^{* *}$ & 13,73 \\
\hline $\begin{array}{l}\text { Psidium cattleianum } \\
\text { Sabine } \\
\text { Oleaceae }\end{array}$ & araçá & Brasil $^{\mathrm{a}}$ & 14,1 & 7,9 & 4 & 0 & 0,17 \\
\hline $\begin{array}{l}\text { Ligustrum japonicum } \\
\text { Thunb. } \\
\text { Paulowniaceae }\end{array}$ & ligustro & Japão $^{b}$ & 21,5 & 5,8 & 19 & 0 & 0,82 \\
\hline $\begin{array}{l}\text { Paulownia tomentosa } \\
\text { (Thunb.) Steud. } \\
\text { Pinaceae }\end{array}$ & kiri & China $^{b}$ & 53,7 & 14,3 & 6 & $1^{*}+1^{* *}$ & 0,26 \\
\hline Pinus spp. & pinus & Hemisfério Norte ${ }^{b}$ & 33,9 & 23,0 & 208 & $4^{*}+2^{* *}$ & 8,95 \\
\hline $\begin{array}{l}\text { Rhamnaceae } \\
\text { Hovenia dulcis Thunb. }\end{array}$ & uva do & Japão, China e Himalaia b & 38,7 & 9,4 & 2 & 0 & 0,09 \\
\hline Rutaceae & Japão & & & & & & \\
\hline $\begin{array}{l}\text { Citrus sp. } \\
\text { Sapindaceae }\end{array}$ & & Ásia ${ }^{c}$ & 53,8 & 9,8 & 2 & 0 & 0,09 \\
\hline $\begin{array}{l}\text { Matayba elaeagnoides } \\
\text { Radlk. }\end{array}$ & camboatá & $\begin{array}{l}\text { Brasil, Uruguai, Argentina } \\
\text { e Paraguai }^{a}\end{array}$ & 28,7 & 7,3 & 3 & 0 & 0,13 \\
\hline Taxodiaceae & & & & & & & \\
\hline $\begin{array}{l}\text { Cryptomeria japonica } \\
\text { D.Don. } \\
\text { Theaceae }\end{array}$ & criptoméria & China e Japão ${ }^{b}$ & 20,7 & 8,6 & 4 & 0 & 0,17 \\
\hline Camellia japonica L. & camélia & China e Japão $^{\text {b }}$ & 20,8 & 6,6 & 1 & 0 & 0,04 \\
\hline
\end{tabular}

${ }^{a}$ Oliveira Filho, 2010; ' Lorenzi et al., 2003; ' Alves e Melo, 2010.

Avaliando os indivíduos presentes, pode-se observar que os mais freqüentes pertencem ao gênero Eucalyptus, com 319 árvores, seguido pelo gênero Pinus, com 208 árvores e pelas espécies de Cupressus lusitanica Mill. (57 indivíduos) e Schinus terebinthifolius Raddi (53 indivíduos).

Dentro do perímetro do CAV, 34 árvores foram danificadas, sendo que 22 foram arrancadas e 12 foram quebradas (Tabela 1). Os danos, de acordo com o teste quiquadrado, não ocorreram de forma aleatória $(p=0,0023)$, sendo dependente das espécies. Isto significa que algumas espécies utilizadas na arborização do local foram susceptíveis aos ventos desta tempestade, enquanto outras não.

As espécies mais danificadas em número de indivíduos foram o eucalipto (nove indivíduos), seguido do cipreste português (sete indivíduos) e do pinus (seis indivíduos). Dos nove indivíduos danificados de Eucalyptus, seis foram arrancados e três quebrados. Porém, pelo teste qui-quadrado, o número de indivíduos que se esperava estar danificados era de 
13,73. Como o número de indivíduos danificados (nove) foi menor que o esperado $(13,73)$, a espécie não é considerada susceptível à tempestade que ocorreu na região. O mesmo ocorreu com o pinus, que obteve número de árvores danificadas (seis) menor que o esperado $(8,95)$.

As espécies que tiverem o numero de árvores danificadas maior do que o esperado para uma distribuição aleatória e, portanto, podem ser consideradas susceptíveis à tempestade ocorrida, foram: Cupressus lusitanica (sete indivíduos danificados, maior que o esperado de 2,45), Mimosa scabrella Benth. (três indivíduos danificados, maior que o esperado de 0,82), Tipuana tipu (Benth.) Kuntze (três indivíduos danificados, maior que o esperado de 0,30), Araucaria angustifolia (Bertol.) Kuntze (dois indivíduos danificados, maior que o esperado de 0,90), Paulownia tomentosa (Thunb.) Steud. (dois indivíduos danificados, maior que o esperado de 0,26) e Senna pendula (Willd.) H.S.Irwin \& Barneby (um indivíduo danificado, maior que o esperado de 0,09 ).

Dos sete indivíduos danificados de Cupressus lusitanica, cinco foram arrancados, inclusive com a raiz, e dois foram quebrados (Figura 1). Os indivíduos arrancados eram, em geral, de médio a grande porte e foi observada a presença de um sistema radicular pequeno. Além disso, segundo Kothiyal et al. (1998), Cupressus lusitanica apresenta madeira fraca e inadequada para usos que requerem resistência mecânica, o que facilita sua quebra. Kothiyal et al. (1998) e Pereira e Higa (2003), estudando a densidade da madeira de Cupressus lusitanica, encontraram baixa densidade $\left(0,415 \mathrm{~g} / \mathrm{cm}^{3}\right)$, o que reforça sua baixa resistência mecânica. 

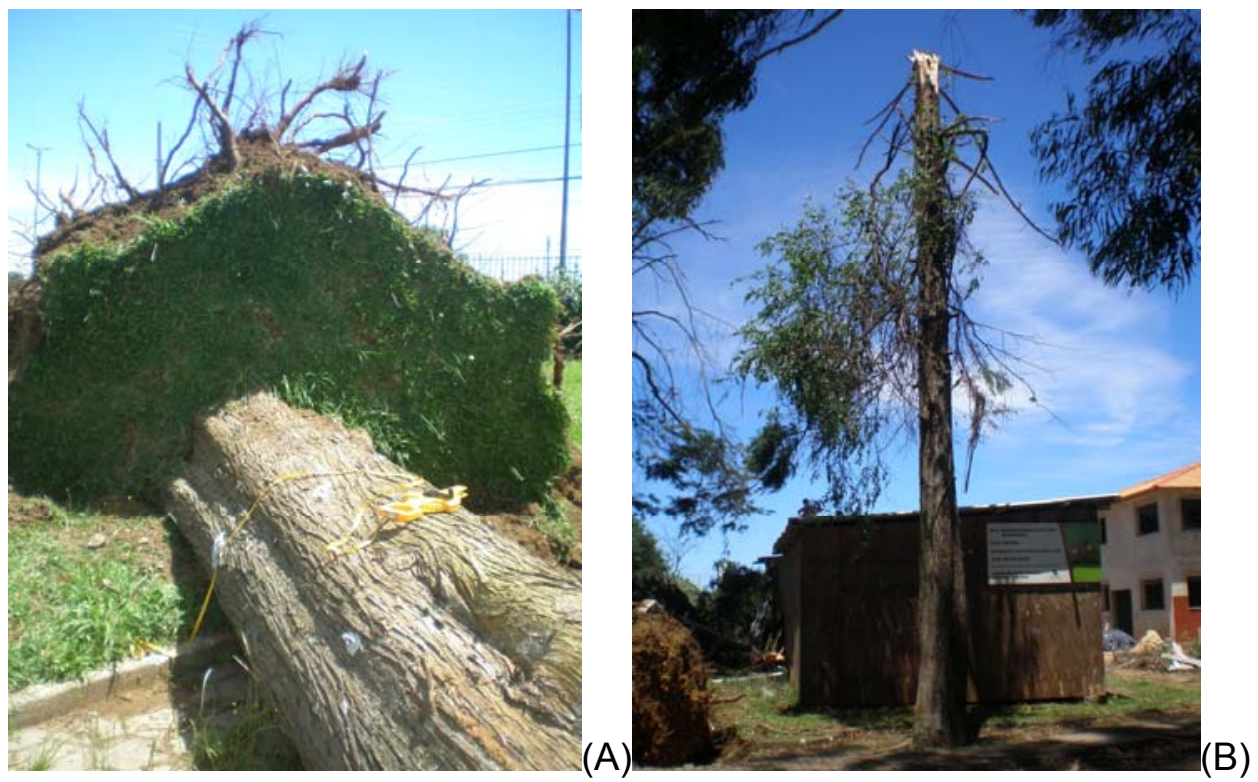

FIGURA 1 - Indivíduos de Cupressus lusitanica no CAV/UDESC arrancados pela raiz (A) e com tronco quebrado (B) após a tempestade ocorrida em Lages, SC, no dia 2 de dezembro de 2009.

Tipuana tipu (Figura 2), outra espécie considerada suscetível aos ventos neste estudo, foi também considerada suscetível à ação de cupins por Bessel (2010), o que também diminui sua resistência mecânica, tornando-a propensa à quebra. Segundo Bessel (2010), é desaconselhado seu plantio em ruas também devido ao seu grande porte.

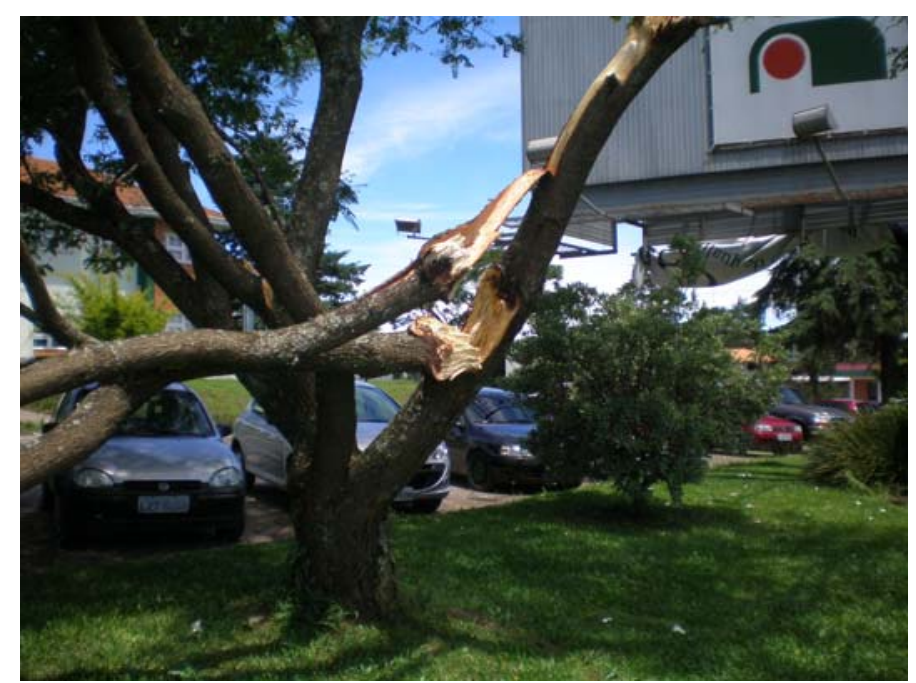

FIGURA 2- Indivíduo de Tipuana tipu no CAV/UDESC com caules quebrados após a tempestade ocorrida em Lages, SC, no dia 2 de dezembro de 2009. 


\section{CONCLUSÕES}

Foram encontrados 791 indivíduos pertencentes a 39 espécies arbóreas no levantamento florístico realizado no CAV/UDESC, sendo que as espécies pertencentes aos gêneros Eucalyptus e Pinus foram as mais abundantes na área.

Apesar de apenas uma pequena proporção das árvores terem sido danificadas $(4,8 \%)$, estas foram responsáveis por um considerável prejuízo material durante a passagem da tempestade com fortes ventos no campus universitário. Além disso, os danos nas árvores não ocorreram de forma aleatória, mas foram dependentes da espécie. De acordo com os resultados encontrados, as espécies Cupressus lusitanica, Mimosa scabrella, Tipuana tipu, Araucaria angustifolia, Paulownia tomentosa e Senna pendula foram consideradas susceptíveis, não sendo indicadas para a arborização urbana de cidades sujeitas a fortes ventos, a fim de se evitar futuras perdas materiais e acidentes.

Vale ressaltar que o presente estudo considerou apenas a ocorrência de um evento de ventos fortes, o que limita a generalização dos resultados, de forma que serão necessárias avaliações futuras após outros eventos climáticos semelhantes para que se possa refinar o conhecimento sobre a suscetibilidade de espécies da arborização urbana.

\section{REFERÊNCIAS BIBLIOGRÁFICAS}

ALVES, P.R B.; MELO, B. Cultura do Citros. Disponível em: http://www.fruticultura.iciag.ufu.br/citros2.htm. Acesso em: 18 de fev. 2010.

BESSEL, L. R. Árvores de rua: cuidados especiais no verão. Disponível em: http://www.sampaonline.com.br/reportagens/arvoresderua2005jan27.htm. Acesso em: 18 de fev. 2010.

BRADSHAW, A.; HUNT, B.; WALMSLEY, T. Trees in the urban landscape: principles and practice. London: E.F.N. Spon, 1995. 272 p.

IBGE - INSTITUTO BRASILEIRO DE GEOGRAFIA E ESTATÍSTICA. Censo demográfico 2007. Disponível em: http://www.ibge.gov.br/home. Acesso em: 15 de dez. 2009. 
KOTHIYAL, V.; NEGI, A.; RAO, R. V.; GOGATE, M. G.; DAKSHINDAS, S. K. Wood quality of eighteen years old Cupressus lusitanica from Maharashtra. Wood Science and Technology, Berlin/Heidelberg, v. 32, n. 2, p. 119-127, abr. 1998.

LEAL, L.; BIONDI, D.; ROCHADELLI, R. Investimentos destinados à arborização de ruas na cidade de Curitiba: uma abordagem a partir da Teoria de Renda da Terra. Scientia Forestalis, Piracicaba, v. 36, n. 78, p. 141-149, jun. 2008.

LORENZI, H.; SOUZA, H. M.; TORRES, M. A. V.; BACHER, L. B. Árvores exóticas no Brasil: madeireiras, ornamentais e aromáticas. Nova Odessa: Instituto Plantarum, 2003. 368 p.

OLIVEIRA, S.; LOPES A. Metodologia de avaliação do risco de queda de árvores devido a ventos fortes, o caso de Lisboa. In: CONGRESSO DA GEOGRAFIA PORTUGUESA, 6. , 2007, Lisboa. Anais... Lisboa, 2007. p. 1-21.

OLIVEIRA-FILHO, A. T. TreeAtlan versão 1.0. Disponível em: http://www.icb.ufmg.br/treeatlan/. Acesso em: 18 de fev. 2010.

PEREIRA, J. C. D.; HIGA, R. C. V. Propriedades da Madeira de Cupressus lusitanica Mill. Comunicado Técnico 107, ISSN 1517-5030, Colombo, 2003.

RANDRUP, T. B.; MCPHERSON, E. G.; COSTELLO, L. R. A review of tree root conflicts with sidewalks, curbs and roads. Urban Ecosystems, Holanda, v. 5, n. 3, p. 209-225, set. 2003.

R DEVELOPMENT CORE TEAM. R: A language and environment for statistical computing. Disponível em: http://www.R-project.org. Acesso em: 15 de dez. 2009.

SETH, M. K. Trees and their economic importance. The Botanical Review, New York, v. 69, n. 4 , p. 321-376, abr. 2004. 
SILVA, M. M. Laudo Meteorológico. EPAGRI (Empresa de Pesquisa Agropecuária e Extensão Rural de Santa Catarina S.A). Setor de previsão de tempo e clima - Epagri/Ciram. Florianópolis, jan. 2010. 